Interval oscillation criteria for second order nonlinear differential equations with nonlinear damping

\author{
Ercan Tunç and Hakan Avci
}




\title{
INTERVAL OSCILLATION CRITERIA FOR SECOND ORDER NONLINEAR DIFFERENTIAL EQUATIONS WITH NONLINEAR DAMPING
}

\author{
ERCAN TUNÇ AND HAKAN AVCI
}

Received March 30, 2011

\begin{abstract}
Using generalized Riccati transformations, we derive new interval oscillation criteria for a class of general type second-order nonlinear differential equations with nonlinear damping. Examples are also given to illustrate the results.
\end{abstract}

2000 Mathematics Subject Classification: 34C10

Keywords: interval oscillation, nonlinear differential equation, nonlinear damping

\section{INTRODUCTION}

In 1836 Sturm introduced the concept of oscillation when he studied the problem of the heat transmission, oscillation theory has been an important area of research in the qualitative theory of ordinary differential equations. Usually a qualitative approach is concerned with the behavior of solutions of a given differential equation and does not seek explicit solutions. Since then, oscillation behavior of solutions to different classes of linear and nonlinear ordinary differential equations have attracted the attention of many researchers.

In this paper we are concerned with the oscillatory behavior of the nonlinear second order differential equation with damping term

$$
\left(r(t) k_{1}\left(x, x^{\prime}\right)\right)^{\prime}+p(t) k_{2}\left(x, x^{\prime}\right) x^{\prime}+q(t) f(x)=0, \quad t \geq t_{0},
$$

where $t_{0} \geq 0$ is a fixed real number, $p, q \in C\left(\left[t_{0}, \infty\right), R\right), r \in C^{1}\left(\left[t_{0}, \infty\right),(0, \infty)\right)$, $f \in C(R, R), k_{1} \in C^{1}\left(R^{2}, R\right)$ and $k_{2} \in C\left(R^{2}, R\right)$. As usual, a function $x:\left[t_{0}, t_{1}\right) \rightarrow$ $(-\infty, \infty), t_{1}>t_{0}$, is called a solution of Eq. (1.1) if $x(t)$ satisfies Eq. (1.1) for all $t \in\left[t_{0}, t_{1}\right)$. In what follows, we always assume that solutions of this equation are continuable, that is, they exist for all $t \geq t_{0}$. A nonconstant continuable solution $x(t)$ of Eq.(1.1) is called proper if $\sup \left\{|x(t)|: t \geq t_{0}\right\}>0$. A proper solution $x(t)$ is called oscillatory if it does not have the largest zero, otherwise it is called nonoscillatory. The equation (1.1) is called oscillatory if all its proper solutions are oscillatory. 
The oscillation problem for Eq. (1.1) and its various particular cases has been the subject of intensive studies in recent years (see, for example ([1-42]) and the references cited therein).

In 2005, Tiryaki and Zafer [30] established several new interval oscillation criteria for Eq. (1.1) by making use of a generalized Riccati substitutions

$$
w(t)=\rho(t)\left[\frac{r(t) k_{1}\left(x(t), x^{\prime}(t)\right)}{x(t)}+g(t)\right]
$$

and

$$
w(t)=\rho(t)\left[\frac{r(t) k_{1}\left(x(t), x^{\prime}(t)\right)}{f(x(t))}+g(t)\right]
$$

where $\rho \in C^{1}\left(\left[t_{0}, \infty\right),(0, \infty)\right)$ and $g \in C^{1}\left(\left[t_{0}, \infty\right), R\right)$. The results in [30] extend, improve, and generalize some known oscillation criteria in the literature. As pointed out earlier Tiryaki and Zafer [29], equation (1.1) deserves attention simply because it includes (as special case) quite a large class of equations in the literature, and therefore more research is necessary to enlarge the families of the functions $k_{1}$ and $k_{2}$. Motivated by the works of Tiryaki and Zafer ([29], [30]), we establish some sufficient conditions which guarantee the oscillatory of all the solutions of Eq. (1.1). We also want to emphasize that the results in this work neither include, nor are included by the results in [30] and are interesting in their own right. At the end, examples are given to illustrate the theoretical analysis of this work.

Let $D=\left\{(t, s): t \geq s \geq t_{0}\right\}$. A function $H \in C(D, R)$ is said to belong to a function class $F$ if

(i) $H(t, t)=0$ for $t \geq t_{0}$ and $H(t, s)>0$ for $t>s \geq t_{0}$;

(ii) $H$ has continuous partial derivatives $\partial H / \partial t$ and $\partial H / \partial s$ satisfying

$$
\begin{aligned}
& \frac{\partial H}{\partial t}(t, s)=h_{1}(t, s) \sqrt{H(t, s)} \quad \text { and } \quad \frac{\partial H}{\partial s}(t, s)=-h_{2}(t, s) \sqrt{H(t, s)}, \\
& \text { where } h_{1}, h_{2} \in L_{l o c}(D, R) .
\end{aligned}
$$

\section{OSCILLATION RESULTS FOR $f(x)$ WITHOUT MONOTONOCITY}

In this section we consider the oscillation of Eq. (1.1) when the function $f(x)$ is not monotone. In this case we shall assume that the following conditions are satisfied:

(A1) $\frac{f(x)}{x} \geq K$ for some constant $K>0$ and all $x \in R \backslash\{0\}$;

(A2) $k_{1}^{2}(u, v) \leq \alpha_{1} v k_{1}(u, v)$ for some constant $\alpha_{1}>0$ and all $(u, v) \in R^{2}$;

(A3) $u v k_{2}(u, v) \geq \alpha_{2} u k_{1}(u, v)$ for some constant $\alpha_{2}>0$ and all $(u, v) \in R^{2}$.

Theorem 1. Let (A1)-(A3) hold. Suppose that there exists an interval $(a, b) \subset$ $\left[t_{0}, \infty\right)$ such that $p(t) \geq 0$ and $q(t) \geq 0$ for all $t \in(a, b)$, and that there exist $c \in(a, b)$, 
$H \in F$ and $\rho \in C^{1}\left(\left[t_{0}, \infty\right),(0, \infty)\right)$ such that

$$
\begin{aligned}
& \frac{1}{H(c, a)} \int_{a}^{c} H(s, a) K \rho(s) q(s) d s+\frac{1}{H(b, c)} \int_{c}^{b} H(b, s) K \rho(s) q(s) d s \\
& >\frac{\alpha_{1}}{4 H(c, a)} \int_{a}^{c} \rho(s) r(s)\left[h_{1}(s, a)+Q_{1}(s) \sqrt{H(s, a)}\right]^{2} d s \\
& +\frac{\alpha_{1}}{4 H(b, c)} \int_{c}^{b} \rho(s) r(s)\left[h_{2}(b, s)-Q_{1}(s) \sqrt{H(b, s)}\right]^{2} d s,
\end{aligned}
$$

where

$$
Q_{1}(s)=\left(\frac{\rho^{\prime}(s)}{\rho(s)}-\frac{\alpha_{2} p(s)}{r(s)}\right) .
$$

Then every solution of Eq. (1.1) has a zero in $(a, b)$.

Proof. For the sake of contradiction, assume that there exits a solution $x(t)$ of Eq. (1.1) such that $x(t) \neq 0$ for all $t \in(a, b)$. Introduce a new function $w$ by

$$
w(t)=\rho(t) \frac{r(t) k_{1}\left(x(t), x^{\prime}(t)\right)}{x(t)}, \quad t \in(a, b) .
$$

Note that $w$ is well-defined because $x(t) \neq 0$ for all $t \in(a, b)$. Differentiating (2.3), we obtain

$$
\begin{aligned}
w^{\prime}(t) & =\frac{\rho^{\prime}(t)}{\rho(t)} w(t)-\rho(t) p(t) \frac{k_{2}\left(x(t), x^{\prime}(t)\right) x^{\prime}(t)}{x(t)}-\rho(t) q(t) \frac{f(x(t))}{x(t)} \\
& -\rho(t) \frac{r(t) k_{1}\left(x(t), x^{\prime}(t)\right) x^{\prime}(t)}{x^{2}(t)} .
\end{aligned}
$$

Using (A1)-(A3) in (2.4), one has

$$
w^{\prime}(t) \leq-K \rho(t) q(t)+\left(\frac{\rho^{\prime}(t)}{\rho(t)}-\frac{\alpha_{2} p(t)}{r(t)}\right) w(t)-\frac{1}{\alpha_{1} \rho(t) r(t)} w^{2}(t) .
$$

Multiplying (2.5) by $H(t, s)$, integrating it with respect to $s$ from $c$ to $t$ for $t \in[c, b)$, and using (i) and (ii), we easily obtain

$$
\begin{aligned}
& \int_{c}^{t} H(t, s) K \rho(s) q(s) d s \\
& \leq-\int_{c}^{t} H(t, s) w^{\prime}(s) d s+\int_{c}^{t} H(t, s) Q_{1}(s) w(s) d s-\int_{c}^{t} \frac{H(t, s) w^{2}(s)}{\alpha_{1} \rho(s) r(s)} d s \\
& =H(t, c) w(c)
\end{aligned}
$$




$$
\begin{aligned}
& -\int_{c}^{t}\left\{H(t, s) \frac{w^{2}(s)}{\alpha_{1} \rho(s) r(s)}+\left[h_{2}(t, s) \sqrt{H(t, s)}-Q_{1}(s) H(t, s)\right] w(s)\right\} d s \\
& =H(t, c) w(c) \\
& -\int_{c}^{t}\left[\sqrt{\left.\frac{H(t, s)}{\alpha_{1} \rho(s) r(s)} w(s)+\frac{1}{2} \sqrt{\alpha_{1} \rho(s) r(s)}\left(h_{2}(t, s)-Q_{1}(s) \sqrt{H(t, s)}\right)\right]^{2} d s}\right. \\
& +\frac{\alpha_{1}}{4} \int_{c}^{t} \rho(s) r(s)\left[h_{2}(t, s)-Q_{1}(s) \sqrt{H(t, s)}\right]^{2} d s \\
& \leq H(t, c) w(c)+\frac{\alpha_{1}}{4} \int_{c}^{t} \rho(s) r(s)\left[h_{2}(t, s)-Q_{1}(s) \sqrt{H(t, s)}\right]^{2} d s .
\end{aligned}
$$

Similarly, multiplying (2.5) by $H(s, t)$ and integrating from $t$ to $c$ for $t \in(a, c]$, one obtains

$$
\begin{aligned}
& \int_{t}^{c} H(s, t) K \rho(s) q(s) d s \\
& \leq-H(c, t) w(c)+\frac{\alpha_{1}}{4} \int_{t}^{c} \rho(s) r(s)\left[h_{1}(s, t)+Q_{1}(s) \sqrt{H(s, t)}\right]^{2} d s .
\end{aligned}
$$

Letting $t \rightarrow a^{+}$in (2.7) and $t \rightarrow b^{-}$in (2.6), dividing (2.6) and (2.7) by $H(b, c)$ and $H(c, a)$, respectively, and then adding them, we have that

$$
\begin{aligned}
& \frac{1}{H(c, a)} \int_{a}^{c} H(s, a) K \rho(s) q(s) d s+\frac{1}{H(b, c)} \int_{c}^{b} H(b, s) K \rho(s) q(s) d s \\
& \leq \frac{\alpha_{1}}{4 H(c, a)} \int_{a}^{c} \rho(s) r(s)\left[h_{1}(s, a)+Q_{1}(s) \sqrt{H(s, a)}\right]^{2} d s \\
& +\frac{\alpha_{1}}{4 H(b, c)} \int_{c}^{b} \rho(s) r(s)\left[h_{2}(b, s)-Q_{1}(s) \sqrt{H(b, s)}\right]^{2} d s,
\end{aligned}
$$

which contradicts the assumption (2.1). The proof is complete.

Thus by Theorem 1, we have the following oscillation result:

Theorem 2. Let (A1)-(A3) hold. Suppose that there exists an interval $(a, b) \subset$ $\left[t_{0}, \infty\right)$ such that $p(t) \geq 0$ and $q(t) \geq 0$ for all $t \in(a, b)$. If, for every $T \geq t_{0}$, there 
exist $H \in F, \rho \in C^{1}\left(\left[t_{0}, \infty\right),(0, \infty)\right)$, and $a, b, c \in R$ such that $T \leq a<c<b$ and (2.1) holds, then every solution of Eq. (1.1) is oscillatory.

Theorem 3. Let (A1)-(A3) hold, and $p(t) \geq 0$ and $q(t) \geq 0$ for all $t \in\left[t_{0}, \infty\right)$. If $\limsup _{t \rightarrow \infty} \int_{l}^{t}\left\{H(s, l) K \rho(s) q(s)-\frac{\alpha_{1} \rho(s) r(s)}{4}\left[h_{1}(s, l)+Q_{1}(s) \sqrt{H(s, l)}\right]^{2}\right\} d s>0$

and

$$
\limsup _{t \rightarrow \infty} \int_{l}^{t}\left\{H(t, s) K \rho(s) q(s)-\frac{\alpha_{1} \rho(s) r(s)}{4}\left[h_{2}(t, s)-Q_{1}(s) \sqrt{H(t, s)}\right]^{2}\right\} d s>0,
$$

for some $H \in F, \rho \in C^{1}\left(\left[t_{0}, \infty\right),(0, \infty)\right)$ and for each $l \geq t_{0}$, where $Q_{1}(s)$ is as in (2.2), then every solution of Eq. (1.1) is oscillatory.

Proof. For any $T \geq t_{0}$, let $a=T$. In (2.8), we choose $l=a$. Then there exists $c>a$ such that

$$
\int_{a}^{c}\left\{H(s, a) K \rho(s) q(s)-\frac{\alpha_{1} \rho(s) r(s)}{4}\left[h_{1}(s, a)+Q_{1}(s) \sqrt{H(s, a)}\right]^{2}\right\} d s>0 .
$$

In (2.9), we choose $l=c$. Then there exists $b>c$ such that

$$
\int_{c}^{b}\left\{H(b, s) K \rho(s) q(s)-\frac{\alpha_{1} \rho(s) r(s)}{4}\left[h_{2}(b, s)-Q_{1}(s) \sqrt{H(b, s)}\right]^{2}\right\} d s>0 .
$$

Combining (2.10) and (2.11), we obtain (2.1). The required conclusion thus follows from Theorem 2. The proof is complete.

Corollary 1. If the inequality in (A3) is actually an equality, we can eliminate the sign condition on $p(t)$ in the above theorems.

Remark 1. If (A3) hold with equality $\left(\alpha_{2}=1\right)$, then Theorem 1,2 and 3 reduce to Theorem 3.3, 3.4 and Corollary 3.2, respectively, obtained by Tiryaki and Zafer [30] with $g(t)=0$ in the substitution (1.2).

Remark 2. If we use the substitution (1.2) instead of (2.3) and the condition (A3) is replaced with

$$
u v k_{2}(u, v) \geq \alpha_{2} k_{1}^{2}(u, v), \alpha_{2}>0,
$$

then Theorem 1, 2 and 3 becomes Theorem 3.1, Corollary 3.1 and Theorem 3.2 of Tiryaki and Zafer [30], respectively. 
Remark 3. We note that the case $p(t) \leq 0$ can be handled in a similar manner by reversing the inequality sign in (A3).

Remark 4. If $k_{1}(u, v)=v, p(t) \equiv 0, \rho(t)=1$ and $f(x)=x$, we also recover Corollary 2.2, Theorem 2.1 and Corollary 2.4 of Kong [12], from Theorem 1, 2 and 3 , respectively.

It is easy to see that when $H(t, s)=H(t-s) \in F, h_{1}(t-s) \equiv h_{2}(t-s)$. From now on, we denote them by $h(t-s)$. Furthermore, the subclass of $F$ containing such $H(t-s)$ will be denoted by $F_{0}$. Applying Theorem 2 to $F_{0}$ we have the following result:

Theorem 4. Suppose that (A1)-(A3) hold, and $p(t) \geq 0$ and $q(t) \geq 0$ for all $t \in$ $(a, b)$. If for each $T \geq t_{0}$, there exist $\rho \in C^{1}\left(\left[t_{0}, \infty\right),(0, \infty)\right), H \in F_{0}$, and real numbers $a, c$ such that $T \leq a<c$ and

$$
\begin{aligned}
& \int_{a}^{c} H(s-a) K[\rho(s) q(s)+\rho(2 c-s) q(2 c-s)] d s \\
& >\frac{\alpha_{1}}{4} \int_{a}^{c}[\rho(s) r(s)+\rho(2 c-s) r(2 c-s)] h^{2}(s-a) d s \\
& +\frac{\alpha_{1}}{2} \int_{a}^{c}\left[\rho(s) r(s) Q_{1}(s)-\rho(2 c-s) r(2 c-s) Q_{2}(s)\right] h(s-a) \sqrt{H(s-a)} d s \\
& +\frac{\alpha_{1}}{4} \int_{a}^{c}\left[\rho(s) r(s) Q_{1}^{2}(s)+\rho(2 c-s) r(2 c-s) Q_{2}^{2}(s)\right] H(s-a) d s
\end{aligned}
$$

where $Q_{2}(s)=\left(\frac{\rho^{\prime}(2 c-s)}{\rho(2 c-s)}-\frac{\alpha_{2} p(2 c-s)}{r(2 c-s)}\right)$, then every solution of Eq. (1.1) is oscillatory.

Proof. Let $c=\frac{a+b}{2}$. Then

$$
H(b-c)=H(c-a)=H\left(\frac{b-a}{2}\right),
$$

and for any $w \in L[a, b]$, one has

$$
\int_{c}^{b} w(s) d s=\int_{a}^{c} w(2 c-s) d s .
$$


Therefore,

$$
\int_{c}^{b} H(b-s) K \rho(s) q(s) d s=\int_{a}^{c} H(s-a) K \rho(2 c-s) q(2 c-s) d s
$$

and

$$
\int_{c}^{b} \rho(s) r(s) h^{2}(b-s) d s=\int_{a}^{c} \rho(2 c-s) r(2 c-s) h^{2}(s-a) d s
$$

Thus, (2.12) yields (2.1), and Eq. (1.1) is oscillatory by Theorem 2.

For different choices of $H(t, s)$, we will obtain different sufficient conditions for oscillatory behavior of solutions of Eq. (1.1). Let $H(t, s)=(t-s)^{\lambda}, t \geq s \geq t_{0}$, where $\lambda>1$ is a constant. Then, we obtain the following useful oscillation criterion.

Corollary 2. Assume that (A1)-(A3) hold, and $p(t) \geq 0$ and $q(t) \geq 0$ for all $t \in$ $\left[t_{0}, \infty\right)$. Then every solution of Eq. (1.1) is oscillatory provided that for each $l \geq t_{0}$ and for some $\lambda>1$, there exists a function $\rho \in C^{1}\left(\left[t_{0}, \infty\right),(0, \infty)\right)$ such that the following two inequalities hold:

$$
\limsup _{t \rightarrow \infty} \frac{1}{t^{\lambda-1}} \int_{l}^{t}(s-l)^{\lambda}\left\{K \rho(s) q(s)-\frac{\alpha_{1} \rho(s) r(s)}{4}\left[\frac{\lambda}{(s-l)}+Q_{1}(s)\right]^{2}\right\} d s>0
$$

and

$$
\limsup _{t \rightarrow \infty} \frac{1}{t^{\lambda-1}} \int_{l}^{t}(t-s)^{\lambda}\left\{K \rho(s) q(s)-\frac{\alpha_{1} \rho(s) r(s)}{4}\left[\frac{\lambda}{(t-s)}-Q_{1}(s)\right]^{2}\right\} d s>0 .
$$

Define

$$
R(t)=\int_{l}^{t} \frac{1}{r(s)} d s, \quad t \geq l \geq t_{0}
$$

and set

$$
H(t, s)=[R(t)-R(s)]^{\lambda}, \quad t \geq t_{0}
$$

where $\lambda>1$ is a constant.

If we choose $\rho(t)=1$, by Theorem 3, we have the following oscillatory criterion.

Theorem 5. Let (A1)-(A3) hold, $p(t) \geq 0$ and $q(t) \geq 0$ for all $t \in\left[t_{0}, \infty\right)$, and $\lim _{t \rightarrow \infty} R(t)=\infty$. Then every solution of Eq. (1.1) is oscillatory provided that for 
each $l \geq t_{0}$ and for some $\lambda>1$, the following two inequalities hold:

$$
\begin{aligned}
& \limsup _{t \rightarrow \infty} \frac{1}{R^{\lambda-1}(t)} \int_{l}^{t}\left\{\left(K q(s)-\frac{\alpha_{1} \alpha_{2}^{2} p^{2}(s)}{4 r(s)}\right)[R(s)-R(l)]^{\lambda}\right. \\
& \left.+\frac{\alpha_{1} \alpha_{2} \lambda p(s)}{2 r(s)}[R(s)-R(l)]^{\lambda-1}\right\} d s>\frac{\alpha_{1} \lambda^{2}}{4(\lambda-1)}
\end{aligned}
$$

and

$$
\begin{aligned}
& \limsup _{t \rightarrow \infty} \frac{1}{R^{\lambda-1}(t)} \int_{l}^{t}\left\{\left(K q(s)-\frac{\alpha_{1} \alpha_{2}^{2} p^{2}(s)}{4 r(s)}\right)[R(t)-R(s)]^{\lambda}\right. \\
& \left.-\frac{\alpha_{1} \alpha_{2} \lambda p(s)}{2 r(s)}[R(t)-R(s)]^{\lambda-1}\right\} d s>\frac{\alpha_{1} \lambda^{2}}{4(\lambda-1)}
\end{aligned}
$$

Proof. Since $H(t, s)=[R(t)-R(s)]^{\lambda}$, it is easy to see that

$$
h_{1}(t, s)=\lambda[R(t)-R(s)]^{(\lambda-2) / 2} \frac{1}{r(t)},
$$

and

$$
h_{2}(t, s)=\lambda[R(t)-R(s)]^{(\lambda-2) / 2} \frac{1}{r(s)} .
$$

Noting that

$$
\begin{aligned}
\int_{l}^{t} r(s) h_{1}^{2}(s, l) d s & =\int_{l}^{t} r(s) \lambda^{2}[R(s)-R(l)]^{\lambda-2} \frac{1}{r^{2}(s)} d s \\
& =\frac{\lambda^{2}}{\lambda-1}[R(t)-R(l)]^{\lambda-1}
\end{aligned}
$$

and

$$
\begin{aligned}
\int_{l}^{t} r(s) h_{2}^{2}(t, s) d s & =\int_{l}^{t} r(s) \lambda^{2}[R(t)-R(s)]^{\lambda-2} \frac{1}{r^{2}(s)} d s \\
& =\frac{\lambda^{2}}{\lambda-1}[R(t)-R(l)]^{\lambda-1}
\end{aligned}
$$

In view of $\lim _{t \rightarrow \infty} R(t)=\infty$, it follows that

$$
\lim _{t \rightarrow \infty} \frac{\alpha_{1}}{4 R^{\lambda-1}(t)} \int_{l}^{t} r(s) h_{1}^{2}(s, l) d s=\frac{\alpha_{1} \lambda^{2}}{4(\lambda-1)}
$$


and

$$
\lim _{t \rightarrow \infty} \frac{\alpha_{1}}{4 R^{\lambda-1}(t)} \int_{l}^{t} r(s) h_{2}^{2}(t, s) d s=\frac{\alpha_{1} \lambda^{2}}{4(\lambda-1)} .
$$

From (2.15) and (2.17) we have that

$$
\begin{aligned}
& \limsup _{t \rightarrow \infty} \frac{1}{R^{\lambda-1}(t)} \int_{l}^{t}\left\{H(s, l) K q(s)-\frac{\alpha_{1} r(s)}{4}\left[h_{1}(s, l)-\frac{\alpha_{2} p(s)}{r(s)} \sqrt{H(s, l)}\right]^{2}\right\} d s \\
& =\limsup _{t \rightarrow \infty} \frac{1}{R^{\lambda-1}(t)} \int_{l}^{t}\left\{[R(s)-R(l)]^{\lambda} K q(s)+\frac{\alpha_{1} \alpha_{2} \lambda p(s)}{2 r(s)}[R(s)-R(l)]^{\lambda-1}\right. \\
& \left.-\frac{\alpha_{1} \alpha_{2}^{2} p^{2}(s)}{4 r(s)}[R(s)-R(l)]^{\lambda}\right\} d s-\lim _{t \rightarrow \infty} \frac{1}{R^{\lambda-1}(t)} \int_{l}^{t} \frac{\alpha_{1}}{4} r(s) h_{1}^{2}(s, l) d s \\
& =\limsup _{t \rightarrow \infty} \frac{1}{R^{\lambda-1}(t)} \int_{l}^{t}\left\{\left(K q(s)-\frac{\alpha_{1} \alpha_{2}^{2} p^{2}(s)}{4 r(s)}\right)[R(s)-R(l)]^{\lambda}\right. \\
& \left.+\frac{\alpha_{1} \alpha_{2} \lambda p(s)}{2 r(s)}[R(s)-R(l)]^{\lambda-1}\right\} d s-\frac{\alpha_{1} \lambda^{2}}{4(\lambda-1)}>0,
\end{aligned}
$$

i.e., (2.8) holds. Similarly, (2.16) implies that (2.9) holds. By Theorem 3, Eq. (1.1) is oscillatory. The proof is complete.

Remark 5. If $k_{1}(u, v)=v, p(t) \equiv 0, \rho(t)=1$ and $f(x)=x$, we recover Theorem 2.2 and Theorem 2.3 of Kong [12], from Theorem 4 and 5, respectively.

\section{OSCILLATION RESULTS FOR $f(x)$ WITH MONOTONOCITY}

We shall assume that the following conditions are satisfied:

(A4) $f(x)$ is differentiable, $x f(x) \neq 0$ and $f^{\prime}(x) \geq L$ for some constant $L>0$ and all $x \in R \backslash\{0\}$;

(A5) $v f(u) k_{2}(u, v) \geq \alpha_{3} f(u) k_{1}(u, v)$ for some $\alpha_{3}>0$ and for all $(u, v) \in R^{2}$.

Theorem 6. Let (A2), (A4) and (A5) hold. Suppose that there exists an interval $(a, b) \subset\left[t_{0}, \infty\right)$ such that $p(t) \geq 0$ for all $t \in(a, b)$, and that there exist $c \in(a, b)$, $H \in F$ and $\rho \in C^{1}\left(\left[t_{0}, \infty\right),(0, \infty)\right)$ such that

$$
\frac{1}{H(c, a)} \int_{a}^{c} H(s, a) \rho(s) q(s) d s+\frac{1}{H(b, c)} \int_{c}^{b} H(b, s) \rho(s) q(s) d s
$$




$$
\begin{aligned}
& >\frac{\alpha_{1}}{4 L H(c, a)} \int_{a}^{c} \rho(s) r(s)\left[h_{1}(s, a)+Q_{3}(s) \sqrt{H(s, a)}\right]^{2} d s \\
& +\frac{\alpha_{1}}{4 L H(b, c)} \int_{c}^{b} \rho(s) r(s)\left[h_{2}(b, s)-Q_{3}(s) \sqrt{H(b, s)}\right]^{2} d s,
\end{aligned}
$$

where

$$
Q_{3}(s)=\left(\frac{\rho^{\prime}(s)}{\rho(s)}-\frac{\alpha_{3} p(s)}{r(s)}\right) .
$$

Then every solution of (1.1) has a zero in $(a, b)$.

Proof. For the sake of contradiction, assume that there exits a solution $x(t)$ of (1.1) such that $x(t) \neq 0$ for all $t \in(a, b)$. Introduce a new function $w$ by

$$
w(t)=\rho(t) \frac{r(t) k_{1}\left(x(t), x^{\prime}(t)\right)}{f(x(t))}, \quad t \in(a, b) .
$$

In view of Eq. (1.1), it follows from (3.3) that

$$
\begin{aligned}
w^{\prime}(t) & =\frac{\rho^{\prime}(t)}{\rho(t)} w(t)-\rho(t) p(t) \frac{k_{2}\left(x(t), x^{\prime}(t)\right) x^{\prime}(t)}{f(x(t))}-\rho(t) q(t) \\
& -\rho(t) \frac{r(t) k_{1}\left(x(t), x^{\prime}(t)\right) f^{\prime}(x(t)) x^{\prime}(t)}{f^{2}(x(t))} .
\end{aligned}
$$

Using (A2), (A4) and (A5) in (3.4), we easily obtain

$$
w^{\prime}(t) \leq-\rho(t) q(t)+\left(\frac{\rho^{\prime}(t)}{\rho(t)}-\frac{\alpha_{3} p(t)}{r(t)}\right) w(t)-\frac{L}{\alpha_{1} \rho(t) r(t)} w^{2}(t) .
$$

The rest of the proof is similar to that of Theorem 1.

Theorem 7. Suppose that (A2), (A4) and (A5) hold, and $p(t) \geq 0$ for all $t \in(a, b)$. If, for every $T \geq t_{0}$, there exist $H \in F, \rho \in C^{1}\left(\left[t_{0}, \infty\right),(0, \infty)\right)$, and $a, b, c \in R$ such that $T \leq a<c<b$ and (3.1) holds, then every solution of Eq. (1.1) is oscillatory.

Theorem 8. Let (A2), (A4) and (A5) hold, and $p(t) \geq 0$ for all $t \in\left[t_{0}, \infty\right)$. If

$$
\limsup _{t \rightarrow \infty} \int_{l}^{t}\left\{H(s, l) \rho(s) q(s)-\frac{\alpha_{1} \rho(s) r(s)}{4 L}\left[h_{1}(s, l)+Q_{3}(s) \sqrt{H(s, l)}\right]^{2}\right\} d s>0
$$

and

$$
\limsup _{t \rightarrow \infty} \int_{l}^{t}\left\{H(t, s) \rho(s) q(s)-\frac{\alpha_{1} \rho(s) r(s)}{4 L}\left[h_{2}(t, s)-Q_{3}(s) \sqrt{H(t, s)}\right]^{2}\right\} d s>0,
$$

for some $H \in F, \rho \in C^{1}\left(\left[t_{0}, \infty\right),(0, \infty)\right)$ and for each $l \geq t_{0}$, then every solution of Eq. (1.1) is oscillatory. 
Corollary 3. If the inequality in (A5) is actually an equality, we can eliminate the sign condition on $p(t)$ in the above theorems.

Remark 6. If (A5) hold with equality $\left(\alpha_{3}=1\right)$, then Theorem 6, 7 and 8 reduce to Theorem 2.3, 2.4 and Corollary 2.2, respectively, obtained by Tiryaki and Zafer [30] with $g(t)=0$ in the substitution (1.3).

Remark 7. If we use the substitution (1.3) instead of (3.3) and the condition (A5) is replaced with

$$
v f(u) k_{2}(u, v) \geq \alpha_{3} k_{1}^{2}(u, v), \alpha_{3}>0,
$$

then Theorem 6, 7 and 8 becomes Theorem 2.1, Corollary 2.1 and Theorem 2.2 of Tiryaki and Zafer [30], respectively.

Remark 8 . We note that the case $p(t) \leq 0$ can be handled in a similar manner by reversing the inequality sign in (A5).

Theorem 9. Suppose that (A2), (A4) and (A5) hold, and $p(t) \geq 0$ for all $t \in(a, b)$. Iffor each $T \geq t_{0}$, there exist $\rho \in C^{1}\left(\left[t_{0}, \infty\right),(0, \infty)\right), H \in F_{0}$, and real numbers $a, c$ such that $T \leq a<c$ and

$$
\begin{aligned}
& \int_{a}^{c} H(s-a)[\rho(s) q(s)+\rho(2 c-s) q(2 c-s)] d s \\
& >\frac{\alpha_{1}}{4 L} \int_{a}^{c}[\rho(s) r(s)+\rho(2 c-s) r(2 c-s)] h^{2}(s-a) d s \\
& +\frac{\alpha_{1}}{2 L} \int_{a}^{c}\left[\rho(s) r(s) Q_{3}(s)-\rho(2 c-s) r(2 c-s) Q_{4}(s)\right] h(s-a) \sqrt{H(s-a)} d s \\
& +\frac{\alpha_{1}}{4 L} \int_{a}^{c}\left[\rho(s) r(s) Q_{3}^{2}(s)+\rho(2 c-s) r(2 c-s) Q_{4}^{2}(s)\right] H(s-a) d s,
\end{aligned}
$$

where $Q_{4}(s)=\left(\frac{\rho^{\prime}(2 c-s)}{\rho(2 c-s)}-\frac{\alpha_{3} p(2 c-s)}{r(2 c-s)}\right)$, then every solution of Eq. (1.1) is oscillatory.

Corollary 4. Assume that (A2), (A4) and (A5) hold, and $p(t) \geq 0$ for all $t \in$ $\left[t_{0}, \infty\right)$. Then every solution of Eq. (1.1) is oscillatory provided that for each $l \geq t_{0}$ and for some $\lambda>1$, there exists a function $\rho \in C^{1}\left(\left[t_{0}, \infty\right),(0, \infty)\right)$ such that the following two inequalities hold:

$$
\limsup _{t \rightarrow \infty} \frac{1}{t^{\lambda-1}} \int_{l}^{t}(s-l)^{\lambda}\left\{\rho(s) q(s)-\frac{\alpha_{1} \rho(s) r(s)}{4 L}\left[\frac{\lambda}{(s-l)}+Q_{3}(s)\right]^{2}\right\} d s>0
$$


and

$$
\limsup _{t \rightarrow \infty} \frac{1}{t^{\lambda-1}} \int_{l}^{t}(t-s)^{\lambda}\left\{\rho(s) q(s)-\frac{\alpha_{1} \rho(s) r(s)}{4 L}\left[\frac{\lambda}{(t-s)}-Q_{3}(s)\right]^{2}\right\} d s>0 .
$$

Remark 9. If $k_{1}(u, v)=v, p(t) \equiv 0, \rho(t)=1$ and $f(x)=x$, we also recover Corollary 2.2, Theorem 2.1 and Corollary 2.4, Theorem 2.2, Theorem 2.3 of Kong [12], from Theorem 6, 7, 8 and 9, Corollary 3, respectively.

Example 1. Consider the equation

$$
\left(\left(1+\cos ^{2} t\right) \frac{x^{\prime}}{1+x^{2}}\right)^{\prime}+\frac{1}{1+x^{2}} x^{\prime}+q(t) f(x)=0
$$

where

$$
\begin{gathered}
k_{1}(u, v)=\frac{v}{1+u^{2}}, \quad k_{2}(u, v)=\frac{1}{1+u^{2}}, \quad f(x)=x\left(1+x^{4}\right), \quad\left(\alpha_{1}=\alpha_{2}=\alpha_{3}=1\right), \\
q(t)= \begin{cases}7(t-3 n), \quad 3 n \leq t \leq 3 n+1 \\
7(-t+3 n+2), & 3 n+1<t \leq 3 n+2 \\
g(t), & 3 n+2<t \leq 3 n+3,\end{cases}
\end{gathered}
$$

$g(t)=-n(3 n+3-t)(t-3 n-2)$ and $n \in N_{0}=\{0,1, \ldots\}$. For any $T \geq 0$, there exists $n \in N_{0}$ such that $3 n \geq T$. Let $a=3 n, c=3 n+1, b=2 c-a, \rho(t)=1$ and $H(t-s)=(t-s)^{2}$. Since $f^{\prime}(x)=1+5 x^{4} \geq 1=L$ and $h(t-s)=2$, we get

$$
\begin{aligned}
& \int_{a}^{c} H(s-a)[q(s)+q(2 c-s)] d s \\
& =\int_{3 n}^{3 n+1}(s-3 n)^{2}[7(s-3 n)+7(3 n+2-s)] d s \\
& =14 \int_{3 n}^{3 n+1}(s-3 n)^{2} d s=14 / 3
\end{aligned}
$$

and

$$
\begin{aligned}
& \frac{\alpha_{1}}{4 L} \int_{a}^{c}[r(s)+r(2 c-s)] h^{2}(s-a) d s \\
& +\frac{\alpha_{1}}{2 L} \int_{a}^{c}[-p(s)+p(2 c-s)] h(s-a) \sqrt{H(s-a)} d s
\end{aligned}
$$




$$
\begin{aligned}
& +\frac{\alpha_{1}}{4 L} \int_{a}^{c}\left[\frac{p^{2}(s)}{r(s)}+\frac{p^{2}(2 c-s)}{r(2 c-s)}\right] H(s-a) d s \\
& =\int_{3 n}^{3 n+1}\left[2+\cos ^{2} s+\cos ^{2}(6 n+2-s)\right] d s \\
& +\frac{1}{4} \int_{3 n}^{3 n+1}\left[\frac{1}{1+\cos ^{2} s}+\frac{1}{1+\cos ^{2}(6 n+2-s)}\right](s-3 n)^{2} d s \\
& \leq \int_{3 n}^{3 n+1} 4 d s+\frac{1}{2} \int_{3 n}^{3 n+1}(s-3 n)^{2} d s=4+\frac{1}{6}=25 / 6<14 / 3 .
\end{aligned}
$$

Hence, (3.6) is oscillatory by Theorem 9. The condition (C2) in Remark 7 is not satisfied. Therefore, the results of [30] can not be used to Eq. (3.6).

If we take $f(x)=x(3+2 \cos x)$ in Eq. (3.6), we find that every solution of (3.6) is oscillatory by Theorem 4 . Since the condition (C1) in Remark 2 is not satisfied, the results of [30] can not be used to Eq. (3.6) with $f(x)=x(3+2 \cos x)$.

Example 2. Consider the equation

$$
\left((2+\sin t) \frac{x^{\prime}}{1+x^{2}}\right)^{\prime}-\frac{4+2 \sin t}{1+x^{2}} x^{\prime}+(2+\sin t) x\left(7+x^{4}\right)=0
$$

where

$$
\begin{gathered}
k_{1}(u, v)=\frac{v}{1+u^{2}}, \quad k_{2}(u, v)=\frac{1}{1+u^{2}}, \quad\left(\alpha_{1}=\alpha_{2}=1\right), \\
f(x)=x\left(7+x^{4}\right), \quad(K=7) .
\end{gathered}
$$

Let $H(t, s)=(t-s)^{2}, \rho(t)=1$. Since $p(t)=-4-2 \sin t<0$ and $\alpha_{1} \alpha_{2} p(t)+$ $r(t)<0$, we see that Eq. (3.7) is oscillatory by Theorem 3 and Remark 3.

\section{ACKNOWLEDGEMENT}

The authors would like to express sincere thanks to the anonymous referee for her/his invaluable corrections, comments and suggestions on the paper.

\section{REFERENCES}

[1] J. W. Baker, "Oscillation theorems for a second order damped nonlinear differential equation," SIAM J. appl. Math., vol. 25, pp. 37-40, 1973.

[2] G. J. Butler, L. H. Erbe, and A. B. Mingarelli, "Riccati techniques and variational principles in oscillation theory for linear systems," Trans. Am. Math. Soc., vol. 303, pp. 263-282, 1987.

[3] M. A. El-Sayed, "An oscillation criterion for a forced second-order linear differential equation," Proc. Am. Math. Soc., vol. 118, no. 3, pp. 813-817, 1993. 
[4] S. R. Grace, "Oscillation theorems for second order nonlinear differential equations with damping," Math. Nachr., vol. 141, pp. 117-127, 1989.

[5] S. R. Grace, "Oscillation criteria for second order differential equations with damping," J. Aust. Math. Soc., Ser. A, vol. 49, no. 1, pp. 43-54, 1990.

[6] S. R. Grace, "Oscillation theorems for nonlinear differential equations of second order," J. Math. Anal. Appl., vol. 171, no. 1, pp. 220-241, 1992.

[7] S. R. Grace and B. S. Lalli, "Oscillation theorems for second order superlinear differential equations with damping," J. Aust. Math. Soc., Ser. A, vol. 53, no. 2, pp. 156-165, 1992.

[8] S. R. Grace, B. S. Lalli, and C. C. Yeh, "Addendum: Oscillation theorems for nonlinear secondorder differential equations with a nonlinear damping term," SIAM J. Math. Anal., vol. 19, no. 5, pp. 1252-1253, 1988.

[9] C. Huang, "Oscillation and nonoscillation for second order linear differential equations," J. Math. Anal. Appl., vol. 210, no. 2, pp. 712-723, 1997.

[10] I. V. Kamenev, "An integral criterion for oscillation of linear differential equations of second order," Math. Notes, vol. 23, pp. 136-138, 1978.

[11] M. Kirane and Y. V. Rogovchenko, "Oscillation results for a second order damped differential equation with nonmonotonous nonlinearity," J. Math. Anal. Appl., vol. 250, no. 1, pp. 118-138, 2000.

[12] Q. Kong, "Interval criteria for oscillation of second-order linear ordinary differential equations," J. Math. Anal. Appl., vol. 229, no. 1, pp. 258-270, 1999.

[13] M. K. Kwong and J. S. W. Wong, "Oscillation and nonoscillation of Hill's equation with periodic damping," J. Math. Anal. Appl., vol. 288, no. 1, pp. 15-19, 2003.

[14] M. K. Kwong and A. Zettl, "Integral inequalities and second order linear oscillation," J. Differ. Equations, vol. 45, pp. 16-33, 1982.

[15] H. J. Li, "Oscillation criteria for second order linear differential equations," J. Math. Anal. Appl., vol. 194, no. 1, pp. 217-234, 1995.

[16] W.-T. Li and R. P. Agarwal, "Interval oscillation criteria for second-order nonlinear differential equations with damping," Comput. Math. Appl., vol. 40, no. 2-3, pp. 217-230, 2000.

[17] W.-T. Li and R. P. Agarwal, "Interval oscillation criteria related to integral averaging technique for certain nonlinear differential equations," J. Math. Anal. Appl., vol. 245, no. 1, pp. 171-188, 2000.

[18] W.-T. Li and R. Agarwal, "Interval oscillation criteria for second-order nonlinear differential equations," Ukr. Math. J. 53, No.9, 1391-1406 (2001) and Ukr. Mat. Zh., vol. 53, no. 9, pp. 1161-1173, 2001.

[19] W.-T. Li and C.-K. Zhong, "Integral averages and interval oscillation of second-order nonlinear differential equations," Math. Nachr., vol. 246-247, pp. 156-169, 2002.

[20] O. G. Mustafa, S. P. Rogovchenko, and Y. V. Rogovchenko, "On oscillation of nonlinear secondorder differential equations with damping term," J. Math. Anal. Appl., vol. 298, no. 2, pp. 604-620, 2004.

[21] A. H. Nasr, "Sufficient conditions for the oscillation of forced super-linear second order differential equations with oscillatory potential," Proc. Am. Math. Soc., vol. 126, no. 1, pp. 123-125, 1998.

[22] C. Philos, "Oscillation theorems for linear differential equations of second order," Arch. Math., vol. 53, no. 5, pp. 482-492, 1989.

[23] S. P. Rogovchenko and Y. V. Rogovchenko, "Oscillation theorems for differential equations with a nonlinear damping term,” J. Math. Anal. Appl., vol. 279, no. 1, pp. 121-134, 2003.

[24] Y. V. Rogovchenko, "Oscillation theorems for second-order equations with damping," Nonlinear Anal., Theory Methods Appl., vol. 41, no. 7-8, pp. A, 1005-1028, 2000.

[25] Y. V. Rogovchenko and F. Tuncay, "Interval oscillation criteria for second order differential equations with damping," Dyn. Syst. Appl., vol. 16, no. 2, pp. 337-343, 2007. 
[26] Y. V. Rogovchenko and F. Tuncay, "Interval oscillation of a second order nonlinear differential equation with a damping term," Discrete Contin. Dyn. Syst., vol. 2007, pp. 883-891, 2007.

[27] Y. G. Sun, “A note on Nasr's and Wong's papers," J. Math. Anal. Appl., vol. 286, no. 1, pp. 363-367, 2003.

[28] Y. G. Sun, "New Kamenev-type oscillation criteria for second-order nonlinear differential equations with damping," J. Math. Anal. Appl., vol. 291, no. 1, pp. 341-351, 2004.

[29] A. Tiryaki and A. Zafer, "Oscillation of second-order nonlinear differential equations with nonlinear damping," Math. Comput. Modelling, vol. 39, no. 2-3, pp. 197-208, 2004.

[30] A. Tiryaki and A. Zafer, "Interval oscillation of a general class of second-order nonlinear differential equations with nonlinear damping," Nonlinear Anal., Theory Methods Appl., vol. 60, no. 1, pp. A, 49-63, 2005.

[31] E. Tunç, "Interval oscillation criteria for certain forced second-order differential equations," Carpathian J. Math., vol. 28, no. 2, pp. 337-344, 2012.

[32] E. Tunç and H. Avc1, "New oscillation theorems for a class of second-order damped nonlinear differential equations," Ukr. Math. J., vol. 63, no. 9, pp. 1441-1457, 2012.

[33] E. Tunç, "A note on the oscillation of second order differential equations with damping," J. Comput. Anal. Appl., vol. 12, no. 2, pp. 444-453, 2010.

[34] J. S. W. Wong, "Second order nonlinear forced oscillations," SIAM J. Math. Anal., vol. 19, no. 3, pp. 667-675, 1988.

[35] J. S. W. Wong, "Oscillation criteria for a forced second-order linear differential equation," J. Math. Anal. Appl., vol. 231, no. 1, pp. 235-240, 1999.

[36] J. S. W. Wong, "Oscillation criteria for second order nonlinear differential equations involving general means," J. Math. Anal. Appl., vol. 247, no. 2, pp. 489-505, 2000.

[37] J. S. W. Wong, "On Kamenev-type oscillation theorems for second-order differential equations with damping," J. Math. Anal. Appl., vol. 258, no. 1, pp. 244-257, 2001.

[38] J. Yan, "Oscillation theorems for second order linear differential equations with damping," Proc. Am. Math. Soc., vol. 98, pp. 276-282, 1986.

[39] Q. Yang, "Interval oscillation criteria for a forced second order nonlinear ordinary differential equations with oscillatory potential," Appl. Math. Comput., vol. 135, no. 1, pp. 49-64, 2003.

[40] A. Zhao, Y. Wang, and J. Yan, "Oscillation criteria for second-order nonlinear differential equations with nonlinear damping," Comput. Math. Appl., vol. 56, no. 2, pp. 542-555, 2008.

[41] Z. Zheng, “Note on Wong's paper," J. Math. Anal. Appl., vol. 274, no. 1, pp. 466-473, 2002.

[42] Z. Zheng, "Oscillation criteria for nonlinear second order differential equations with damping," Acta Math. Hung., vol. 110, no. 3, pp. 241-252, 2006.

\section{Authors' addresses}

\section{Ercan Tunç}

Gaziosmanpaşa University, Department of Mathematics, Faculty of Arts and Science, 60250, Tokat, Turkey

E-mail address: ercantunc72@yahoo.com

\section{Hakan Aveı}

Ondokuz Mayis University, Department of Mathematics, Faculty of Arts and Sciences, Samsun, Turkey

E-mail address: hakanav@omu.edu.tr 